\title{
DEGREE BOUND FOR SEPARATING INVARIANTS OF ABELIAN GROUPS
}

\author{
M. DOMOKOS
}

\begin{abstract}
It is proved that the universal degree bound for separating polynomial invariants of a finite abelian group (in non-modular characteristic) is strictly smaller than the universal degree bound for generators of polynomial invariants, unless the goup is cyclic or is the direct product of $r$ even order cyclic groups where the number of two-element direct factors is not less than the integer part of the half of $r$. A characterization of separating sets of monomials is given in terms of zero-sum sequences over abelian groups.
\end{abstract}

\section{INTRODUCTION}

Let $G$ be a finite group and $\mathbb{F}$ an algebraically closed field. A $G$-module is a finite dimensional $\mathbb{F}$-vector space $V$ endowed with an action of $G$ on $V$ via linear transformations. In other words, the $G$-module consists of the pair $(V, \rho)$ where $\rho$ is a group homomorphism $G \rightarrow G L(V)$. The coordinate ring $\mathcal{O}(V)$ of $V$ contains the subalgebra

$$
\mathcal{O}(V)^{G}:=\{f \in \mathcal{O}(V): f(g v)=f(v) \quad \forall v \in V, \forall g \in G\}
$$

of $G$-invariants. For $f \in \mathcal{O}(V)$ and $g \in G$ write $g \cdot f \in \mathcal{O}(V)$ for the function $v \mapsto$ $f\left(g^{-1} v\right)$. This way we get an action of $G$ on $\mathcal{O}(V)$ via $\mathbb{F}$-algebra automorphisms, and $\mathcal{O}(V)^{G}=\{f \in \mathcal{O}(V): g \cdot f=f \quad \forall g \in G\}$. Choosing a basis $x_{1}, \ldots, x_{k}$ in the dual space $V^{*}$ of $V$, the coordinate $\operatorname{ring} \mathcal{O}(V)$ is identified with the polynomial algebra $\mathbb{F}\left[x_{1}, \ldots, x_{k}\right]$, on which $G$ acts via linear substitutions of the variables.

Following Definition 2.3.8 in [5], we call subset $S \subset \mathcal{O}(V)^{G}$ a separating set of invariants if whenever for $v, w \in V$ we have $f(v)=f(w)$ for all $f \in S$, then $h(v)=h(w)$ for all $h \in \mathcal{O}(V)^{G}$. Clearly if $v$ and $w$ belong to the same $G$-orbit in $V$, then $h(v)=h(w)$ holds for all $h \in \mathcal{O}(V)^{G}$. It is well known that the finiteness of $G$ implies the converse as well: if $v$ and $w$ have different $G$-orbits, then there exists an $h \in \mathcal{O}(V)^{G}$ with $h(v) \neq h(w)$. So $S \subset O(V)^{G}$ is a separating set if and only if for any $v, w \in V$ with $G v \neq G w$ there is an $f \in S$ such that $f(v) \neq f(w)$. For a survey on separating sets of invariants see [19].

Since the $G$-action preserves the standard grading on $\mathbb{F}\left[x_{1}, \ldots, x_{k}\right]$, the algebra $\mathcal{O}(V)^{G}$ is generated by homogeneous elements. Write $\beta(G, V)$ (respectively $\left.\beta_{\text {sep }}(G, V)\right)$ for the minimal positive integer $k$ such that $\mathcal{O}(V)^{G}$ contains a generating set (respectively separating set) consisting of homogeneous elements of degree

2010 Mathematics Subject Classification. 13A50, 11B75, $20 \mathrm{~K} 01$.

Key words and phrases. Separating invariants, zero-sum sequences, Noether number, Davenport constant.

This research was partially supported by OTKA K101515. 
at most $k$. Moreover, set

$$
\beta(G):=\sup _{V}\{\beta(G, V)\} \quad \text { and } \quad \beta_{\text {sep }}(G):=\sup _{V}\left\{\beta_{\text {sep }}(G, V)\right\}
$$

where the supremum above is taken over all $G$-modules $V$. The number $\beta_{\text {sep }}(G)$ was introduced and studied in 20 , inspired by the number $\beta(G)$ first appearing in [26]. Obviously $\beta_{\text {sep }}(G, V) \leq \beta(G, V)$ and hence $\beta_{\text {sep }}(G) \leq \beta(G)$. When the characteristic of $\mathbb{F}$ does not divide the group order $|G|$, we have $\beta(G) \leq|G|$ (see 23. for $\operatorname{char}(\mathbb{F})=0$ and [10, 11] for positive non-modular characteristic). One nice feature of $\beta_{\text {sep }}(G)$ is that the inequality $\beta_{\text {sep }}(G) \leq|G|$ holds also in the modular case $\operatorname{char}(\mathbb{F})|| G \mid$ as well, see Corollary 3.9.14 in [5]. In comparison we mention that when $\operatorname{char}(\mathbb{F})$ divides $|G|$ we have $\beta(G)=\infty$ by 25 . However, as far as we know, not much is said in the literature about the following question:

Question 1.1. Is $\beta_{\text {sep }}(G)$ typicallly strictly smaller than $\beta(G)$ in the non-modular case $\operatorname{char}(\mathbb{F}) \nmid|G|$ ?

A difficulty in answering Question 1.1 is that the exact value of the Noether number is known only for a very limited class of groups, see for example [2], [3], 4]. It is shown in [1] that for the non-abelian semidirect product $C_{p} \rtimes C_{3}$ (where $p$ is a prime) and $\operatorname{char}(\mathbb{F})=0$ we have $\beta\left(C_{p} \rtimes C_{3}\right)=p+2$ whereas $\beta_{\text {sep }}\left(C_{p} \rtimes C_{3}\right)=p+1$.

In the present paper we shall deal with abelian groups. Our main result Theorem 3.10 implies that for abelian groups the answer to Question 1.1 is yes. More precisely, Corollary 3.11 asserts that when $G$ is abelian, $\beta_{\text {sep }}(G)=\beta(G)$ implies that $G$ is cyclic or $G$ is the direct product of $r$ cyclic groups of even order, where at least $\left\lfloor\frac{r}{2}\right\rfloor$ of the cyclic factors has order 2 .

A interesting special feature of the case of abelian groups is that the investigation of separating invariants can be tied up with the theory of zero-sum sequences over abelian groups. Given a finite abelian group $G$ (written additively) and an ordered sequence $a_{1}, \ldots, a_{k}$ of elements of $G$ (repetition is allowed) set

$$
\mathcal{G}\left(a_{1}, \ldots, a_{k}\right):=\left\{\left(m_{1}, \ldots, m_{k}\right) \in \mathbb{Z}^{k}: \sum m_{i} a_{i}=0 \in G\right\} .
$$

This is a subgroup of the free abelian group $\mathbb{Z}^{k}$. It contains the submonoid

$$
\mathcal{B}\left(a_{1}, \ldots, a_{k}\right):=\mathbb{N}_{0}^{k} \cap \mathcal{G}\left(a_{1}, \ldots, a_{k}\right) .
$$

Denote by $e_{i}$ the $i$ th standard basis vector in $\mathbb{Z}^{k}$. Clearly ord $\operatorname{ord}_{G}\left(a_{i}\right) e_{i} \in \mathcal{B}\left(a_{1}, \ldots, a_{k}\right)$, where $\operatorname{ord}_{G}\left(a_{i}\right)$ is the order of $a_{i}$ in $G$. Since for any $m \in \mathcal{G}\left(a_{1}, \ldots, a_{k}\right)$ there exist non-negative integers $t_{1}, \ldots, t_{k} \in \mathbb{N}_{0}$ with $m+\sum t_{i} \operatorname{ord}_{G}\left(a_{i}\right) e_{i} \in \mathcal{B}\left(a_{1}, \ldots, a_{k}\right)$, it follows that $\mathcal{G}\left(a_{1}, \ldots, a_{k}\right)$ is the quotient group of the monoid $\mathcal{B}\left(a_{1}, \ldots, a_{k}\right)$. In particular, the abelian group $\mathcal{G}\left(a_{1}, \ldots, a_{k}\right)$ is generated by its submonoid $\mathcal{B}\left(a_{1}, \ldots, a_{k}\right)$. In the special case when $a_{1}, \ldots, a_{k}$ are distinct and $\left\{a_{1}, \ldots, a_{k}\right\}=G$, we recover the monoid $\mathcal{B}(G)$ of zero-sum sequences over $G$, a well studied object in arithmetic combinatorics. In particular, the Davenport constant $\mathrm{D}(G)$ is defined as the maximal length of an atom in the monoid $\mathcal{B}(G)$, where for $s \in \mathcal{B}(G) \subset \mathbb{N}_{0}^{|G|}$ the length of $s$ is $|s|=\sum_{g \in G} s_{g}$. More generally, the study of the monoid $\mathcal{B}\left(G_{0}\right)$ of zero-sum sequences over an arbitrary subset $G_{0}$ of $G$ has an extensive literature, see Proposition 2.5.6 in [15] for the first abstract algebraic properties of the monoid $\mathcal{B}\left(G_{0}\right)$, or 24] for recent combinatorial work on $\mathrm{D}\left(G_{0}\right)$ (for some very special subset $G_{0}$ ). 
From now on we assume that $G$ is a finite abelian group and the characteristic of the base field $\mathbb{F}$ does not divide $|G|$. Then $V$ decomposes as a direct sum

$$
V=V_{1} \oplus \cdots \oplus V_{k}
$$

of 1-dimensional $G$-modules. Accordingly the variables in $\mathcal{O}(V)=\mathbb{F}\left[x_{1}, \ldots, x_{k}\right]$ will be chosen to be $G$-eigenvectors, so there exist characters $\chi_{i} \in \widehat{G}=\operatorname{hom}\left(G, \mathbb{F}^{\times}\right)$such that $g \cdot x_{i}=\chi_{i}(g) x_{i}$ for $i=1, \ldots, k$. For $m \in \mathbb{N}_{0}^{k}$ write $x^{m}=x_{1}^{m_{1}} \cdots x_{k}^{m_{k}}$. Each monomial spans a $G$-invariant subspace in $\mathcal{O}(V)$, and $g \cdot x^{m}=\left(\prod_{i=}^{k} \chi_{i}(g)^{m_{i}}\right) x^{m}$. It follows that $\mathcal{O}(V)^{G}$ is spanned by $G$-invariant monomials, namely

$$
\mathcal{O}(V)^{G}=\bigoplus_{m \in \mathcal{B}\left(\chi_{1}, \ldots, \chi_{k}\right)} \mathbb{F} x^{m}
$$

Note that here we use the notation introduced in the above paragraph for the finite abelian group $\widehat{G}$ which is a isomorphic to $G$. A consequence of (1) is the equality

$$
\beta(G)=\mathrm{D}(G)
$$

which was used in 26, and later in 9 or in [4. In view of the above connection between the Noether number $\beta(G)$ and the Davenport constant $\mathrm{D}(G)$ it is natural to ask for the meaning of $\beta_{\text {sep }}(G)$ in terms of zero-sum sequences. This is the second motivation of the present paper. In Theorem 2.1] we provide a characterization of separating sets of monomials and zero-sum sequences over $G$, yielding a characterization of $\beta_{\text {sep }}(G)$ purely in terms of zero-sum sequences over $G$ (see Corollary 2.6). This is done in Section 2, and Corollary 2.6 is used in Section 3 to derive our main result Theorem 3.10 .

We finish the introduction by mentioning some prior works related to separating invariants of finite diagonal groups. Namely, a separating set of monomials in $\mathcal{O}(V)^{G}$ is constructed in Proposition 5 of 22 . An algorithm to produce invariant monomials that generate the field of rational invariants is described in 18 . The focus of present paper is on degree bounds for separating invariants, and therefore it is sufficient to deal with invariant monomials. A different current research direction is the study of the minimal cardinality of a separating system, see for example $[\underline{8}$.

\section{Characterization of Separating Sets of monomials}

Let $G$ be a finite abelian group, and let $V=V_{1} \oplus \cdots \oplus V_{k}$ be a $k$-dimensional $G$ module as in Section 1, so $\mathcal{O}(V)=\mathbb{F}\left[x_{1}, \ldots, x_{k}\right]$ with $g \cdot x_{i}=\chi_{i}(g) x_{i}$ for $i=1, \ldots, k$. For $m \in \mathbb{N}_{0}^{k} \operatorname{set} \operatorname{supp}(m):=\left\{i \in\{1, \ldots, k\}: m_{i} \neq 0\right\} \subset\{1, \ldots, k\}$. Similarly, when $x^{m}$ is a monomial, we shall use the notation $\operatorname{supp}\left(x^{m}\right)$ for $\operatorname{supp}(m)$. Given a subset $J \subset\{1, \ldots, k\}$ and a set $M \subset \mathbb{N}_{0}^{k}$ we write $M_{J}:=\{m \in M: \operatorname{supp}(m) \subset J\}$.

The Helly dimension $\kappa(G)$ of $G$ was defined in [6] as the minimal positive integer $k$ such that any set of cosets in $G$ with empty intersection contains a subset of at most $k$ cosets with empty intersection. It was shown in [7] that $\kappa(G)$ is one bigger than the minimal number of generators of the finite abelian group $G$ (the rank of $G)$.

Theorem 2.1. For a subset $M \subset \mathcal{B}\left(\chi_{1}, \ldots, \chi_{k}\right)$ the following are equivalent:

(i) $\left\{x^{m}: m \in M\right\}$ is a separating subset in $\mathcal{O}(V)^{G}$.

(ii) For all subsets $\left\{j_{1}<\cdots<j_{s}\right\}=J \subset\{1, \ldots, k\}$, the abelian group $\mathcal{G}\left(\chi_{j_{1}}, \ldots, \chi_{j_{s}}\right)$ is generated by $M_{J}$. 
(iii) For all subsets $\left\{j_{1}<\cdots<j_{s}\right\}=J \subset\{1, \ldots, k\}$ with $|J| \leq \kappa(G)$, the abelian group $\mathcal{G}\left(\chi_{j_{1}}, \ldots, \chi_{j_{s}}\right)$ is generated by $M_{J}$.

The proof will be split into a couple of statements. Consider the $G$-module direct summand $V_{J}:=\bigoplus_{j \in J} V_{j}$ of $V$, where $J \subset\{1, \ldots, k\}$. Its coordinate ring $\mathcal{O}\left(V_{J}\right)$ is an algebra retract of $\mathcal{O}(V)$ : it is the subalgebra generated by the variables $\left\{x_{j}: j \in J\right\}$. For $v \in V$ we write $v_{J}$ for the component of $V$ in the direct summand $V_{J}$ of $V$. The statement and proof of Lemma 2.2 below remain valid when the finite group $G$ is not assumed to be abelian and the direct summands $V_{j}$ are not assumed to be 1-dimensional.

Lemma 2.2. Assume that $k \geq \kappa(G)$ and for all $J \subset\{1, \ldots, k\}$ with $|J|=\kappa(G)$ we are given a separating subset $S_{J}$ in $\mathcal{O}\left(V_{J}\right)^{G}$. Then their union $S:=\bigcup_{J} S_{J}$ is a separating subset in $\mathcal{O}(V)^{G}$.

Proof. Suppose that for $v, w \in V$ we have $f(v)=f(w)$ for all $f \in S$. Then in particular, for a fixed $J \subset\{1, \ldots, k\}$ with $|J|=\kappa(G)$ we have $f\left(v_{J}\right)=f\left(w_{J}\right)$ for all $f \in S_{J}$. Since $S_{J}$ is a separating subset in $\mathcal{O}\left(V_{J}\right)^{G}$, we conclude that $G v_{J}=G w_{J}$. This holds for all $J \subset\{1, \ldots, k\}$ with $|J|=\kappa(G)$, hence by Lemma 4.1 in [7] we get that $G v=G w$.

Proposition 2.3. Let $M$ be a subset of $\mathcal{B}\left(\chi_{1}, \ldots, \chi_{k}\right)$ such that for all $J=\left\{j_{1}<\right.$ $\left.\cdots<j_{s}\right\} \subset\{1, \ldots, k\}$ the abelian group $\mathcal{G}\left(\chi_{j_{1}}, \cdots, \chi_{j_{s}}\right)$ is generated by $M_{J}$. Then $\left\{x^{m}: m \in M\right\}$ is a separating set in $\mathcal{O}(V)^{G}$.

Proof. Take $v, w \in V$ such that $x^{m}(v)=x^{m}(w)$ for all $m \in M$. The assumption says in particular that $M_{\{j\}}$ generates $\mathcal{G}\left(\chi_{j}\right)$ for $j=1, \ldots, d$. Since $\mathcal{G}\left(\chi_{j}\right)$ is the subgroup of $\mathbb{Z}$ generated by $\operatorname{ord}_{\widehat{G}}\left(\chi_{i}\right)$, it follows that some positive power of $x_{j}$ belongs to $\left\{x^{m}: m \in M\right\}$. Thus $x_{j}(v)=0$ if and only if $x_{j}(w)=0$, so $\operatorname{supp}(v)=$ $\operatorname{supp}(w)=: J$. Take an arbitrary $G$-invariant monomial $x^{n}$. If $\operatorname{supp}(n) \nsubseteq J$, then $x^{n}(v)=0=x^{n}(w)$. Otherwise $\operatorname{supp}(n) \subseteq J=\left\{j_{1}<\cdots<j_{s}\right\}$. Since $M_{J}$ generates $\mathcal{G}\left(\chi_{j_{1}}, \ldots, \chi_{j_{s}}\right)$, there exist $u_{1}, \ldots, u_{k}, t_{1}, \ldots, t_{l} \in M_{J}$ such that $n=$ $u_{1}+\cdots+u_{k}-t_{1}-\cdots-t_{l} \in \mathbb{Z}^{s}$, implying $x^{n} x^{t_{1}} \ldots x^{t_{l}}=x^{u_{1}} \ldots x^{u_{k}}$. By our assumption on $v, w$ we have $u_{i}(v)=u_{i}(w)$ for $i=1, \ldots, k$ and $t_{i}(v)=t_{i}(w) \neq 0$ for $i=1, \ldots, l$. It follows that

$$
x^{n}(v)=\frac{x^{u_{1}}(v) \ldots x^{u_{k}}(v)}{x^{t_{1}}(v) \ldots x^{t_{l}}(v)}=\frac{x^{u_{1}}(w) \ldots x^{u_{k}}(w)}{x^{t_{1}}(w) \ldots x^{t_{l}}(w)}=x^{n}(w) .
$$

Thus we proved that $x^{n}(v)=x^{n}(w)$ holds for an arbitrary $G$-invariant monomial $x^{n}$, implying in turn that $h(v)=h(w)$ for any $h \in \mathcal{O}(V)^{G}$.

Proposition 2.4. Let $M$ be a subset of $\mathcal{B}\left(\chi_{1}, \ldots, \chi_{k}\right)$ such that $\left\{x^{m}: m \in M\right\}$ is a separating set in $\mathcal{O}(V)^{G}$. Then the abelian group $\mathcal{G}\left(\chi_{1}, \ldots, \chi_{k}\right)$ is generated by $M$.

Proof. Since $e_{i} \in V$ can be separated from 0 by $x^{m}$ for some $m \in M$, a positive power $x_{i}^{n_{i}}$ belongs to $\left\{x^{m}: m \in M\right\}$ for each $i=1, \ldots, d$. Obviously it is sufficient to prove the statement when $G$ acts faithfully on $V$, so $G \subset G L(V)$ and hence $\widehat{G}=\left\langle\chi_{1}, \ldots, \chi_{k}\right\rangle$. On the other hand $\left\langle\chi_{1}, \ldots, \chi_{k}\right\rangle \cong \mathbb{Z}^{k} / \mathcal{G}\left(\chi_{1}, \ldots, \chi_{k}\right)$, as $\mathcal{G}\left(\chi_{1}, \ldots, \chi_{k}\right)$ was defined as the kernel of the natural surjection $\mathbb{Z}^{k} \rightarrow \widehat{G}$ with $e_{i} \mapsto \chi_{i}$. Denote by $H$ the abelian group $\mathbb{Z}^{k} / \mathbb{Z} M$. This group is finite, as $n_{i} e_{i} \in M$, hence it is isomorphic to its character group $\widehat{H}$. Therefore we may choose generators 
$\psi_{1}, \ldots, \psi_{k} \in \widehat{H}$ such that the natural surjection $\mathbb{Z}^{k} \rightarrow \widehat{H}, e_{i} \mapsto \psi_{i}(i=1, \ldots, d)$ has kernel $M \mathbb{Z}$. For $h \in H$ let $\rho(h) \in G L(V)$ be the linear transformation given by $\rho(h) \xi_{i}=\psi_{i}\left(h^{-1}\right) \xi_{i}$ where $\xi_{i}$ spans the summand $V_{i}$ in $V=\bigoplus_{i=1}^{k} V_{i}$. Then $\rho: H \rightarrow G L(V)$ is an injective group homomorphism. Note that for any $q \in \mathbb{N}_{0}^{k}$ we have $x^{q}\left(\rho(h)\left(\xi_{1}+\cdots+\xi_{k}\right)\right)=\prod_{i=1}^{k} \psi_{i}\left(h^{-1}\right)^{q_{i}}$. By the choice of $\psi_{i}$, for any $m \in M$ and any $h \in H$ and we have $\prod_{i=1}^{k} \psi_{i}\left(h^{-1}\right)^{m_{i}}=1$. Therefore we have

$$
x^{m}\left(\rho(h)\left(\xi_{1}+\cdots+\xi_{k}\right)\right)=1
$$

for all $m \in M$ and $h \in H$. On the other hand $x^{m}\left(\xi_{1}+\cdots+\xi_{k}\right)=1$ as well. Since $\left\{x^{m}: m \in M\right\}$ is a separating set in $\mathcal{O}(V)^{G}$, we conclude that the $H$-orbit of $\xi_{1}+\cdots+\xi_{k}$ is contained in the $G$-orbit of $\xi_{1}+\cdots+\xi_{k}$. Thus for each $h \in H$ there exists a $g \in G$ such that $\rho(h)\left(\xi_{1}+\cdots+\xi_{k}\right)=g\left(\xi_{1}+\cdots+\xi_{k}\right)$, implying in turn that $\rho(h) \xi_{i}=g \xi_{i}$ for each basis vector $\xi_{i} \in V$, and hence $\rho(h)=g$. So we have $H \cong \rho(H) \subset G \subset G L(V)$. Therefore $\psi_{i}=\chi_{i} \circ \rho$ for $i=1, \ldots, k$, and it follows that the natural surjection $\mathbb{Z}^{k} \rightarrow \widehat{H}, e_{i} \mapsto \psi_{i}$ factors through the natural surjection $\mathbb{Z}^{k} \rightarrow \widehat{G}, e_{i} \mapsto \chi_{i}$, so $\mathbb{Z} M \supset \mathcal{G}\left(\chi_{1}, \ldots, \chi_{k}\right)$. Now as $M$ was a subset of $\mathcal{B}\left(\chi_{1}, \ldots, \chi_{k}\right) \subset \mathcal{G}\left(\chi_{1}, \ldots, \chi_{k}\right)$, the reverse inclusion $\mathbb{Z} M \subset \mathcal{G}\left(\chi_{1}, \ldots, \chi_{k}\right)$ also holds, forcing the equality $\mathbb{Z} M=\mathcal{G}\left(\chi_{1}, \ldots, \chi_{k}\right)$.

Lemma 2.5. If $\left\{x^{m}: m \in M\right\}$ is a separating set in $\mathcal{O}(V)^{G}$, then for all subsets $J \subset\{1, \ldots, k\}$ the monomials $\left\{x^{m}: m \in M_{J}\right\}$ constitute a separating set in $\mathcal{O}\left(V_{J}\right)^{G}$.

Proof. We claim that if $S$ is a separating set in $\mathcal{O}(V)^{G}$, then its restriction $\left\{\left.f\right|_{V_{J}}: f \in\right.$ $S\}$ to $V_{J}$ is a separating set in $\mathcal{O}\left(V_{J}\right)^{G}$. Indeed, suppose $h(v) \neq h(w)$ for some $v, w \in V_{J}$ and $h \in \mathcal{O}\left(V_{J}\right)^{G}$. Since the algebra $\mathcal{O}\left(V_{J}\right)^{G}$ is contained in $\mathcal{O}(V)^{G}$, it follows that there exists an $f \in S$ with $f(v) \neq f(w)$, so $\left.f\right|_{V_{J}}$ separates $v$ and $w$. This proves the claim. Now observe that if $m$ does not belong to $M_{J}$, then the monomial $x^{m}$ vanishes identically on $V_{J}$. Consequently the restriction to $V_{J}$ of $\left\{x^{m}: m \in M\right\}$ is contained in $\left\{x^{m}: m \in M_{J}\right\} \cup\{0\}$, and our statement follows.

Proof of Theorem [2.1. (i) $\Rightarrow$ (ii): Suppose that $\left\{x^{m}: m \in M\right\}$ is a separating set in $\mathcal{O}(V)^{G}$, and take a subset $J=\left\{j_{1}<\cdots<j_{s}\right\} \subset\{1, \ldots, k\}$. By Lemma 2.5 $\left\{x^{m}: m \in M_{J}\right\}$ is a separating set in $\mathcal{O}\left(V_{J}\right)^{G}$. Applying Proposition 2.4 for $V_{J}$ and $M_{J}$ we conclude that the abelian group $\mathcal{G}\left(\chi_{j_{1}}, \ldots, \chi_{j_{s}}\right)$ is generated by $M_{J}$.

(ii) $\Rightarrow$ (iii): Trivial.

(iii) $\Rightarrow$ (i): Suppose that (iii) holds. Then for any subset $J=\left\{j_{1}<\cdots<\right.$ $\left.j_{s}\right\} \subset\{1, \ldots, k\}$ with $s=|J| \leq \kappa(G)$, the set $M_{J}$ generates the abelian group $\mathcal{G}\left(\chi_{j_{1}}, \ldots, \chi_{j_{s}}\right)$, hence by Proposition $2.3\left\{x^{m}: m \in M_{J}\right\}$ is a separating set in $\mathcal{O}\left(V_{J}\right)^{G}$. If $d \leq \kappa(G)$, then we may take $J=\{1, \ldots, k\}$ and we are done. Otherwise the union of the $\left\{x^{m}: m \in M_{J}\right\}$ as $J$ ranges over the subsets of $\{1, \ldots, k\}$ of size $\kappa(G)$ is a separating set in $\mathcal{O}(V)^{G}$ by Lemma 2.2.

Corollary 2.6. The number $\beta_{\mathrm{sep}}(G)$ is the minimal positive integer $d$ such that for any positive integer $s \leq \kappa(G)$ and any finite sequence $a_{1}, \ldots, a_{s}$ of distinct elements of $G$ the abelian group $\mathcal{G}\left(a_{1}, \ldots, a_{s}\right)$ is generated by $\left\{m \in \mathcal{B}\left(a_{1}, \ldots, a_{s}\right):|m| \leq d\right\}$.

Proof. For a finite abelian group $H$ denote by $\delta(H)$ (respectively $\left.\delta_{0}(H)\right)$ the minimal positive integer $d$ such that for any $s \leq \kappa(H)$ and any sequence $a_{1}, \ldots, a_{s}$ of not necessarily distinct (respectively distinct) elements of $H$ the group $\mathcal{G}\left(a_{1}, \ldots, a_{s}\right)$ is 
generated by the $m \in \mathcal{B}\left(a_{1}, \ldots, a_{s}\right)$ with $|m| \leq d$. Obviously $\delta_{0}(H) \leq \mathrm{D}(H) \leq|H|$. We claim that $\delta(H)=\delta_{0}(H)$. Indeed, the inequality $\delta_{0}(H) \leq \delta(H)$ holds by definition of $\delta$ and $\delta_{0}$. To see the reverse inequality take an arbitrary sequence $a_{1}, \ldots, a_{s} \in H, s \leq \kappa(H)$. By induction on $s$ we show that $\mathcal{G}\left(a_{1}, \ldots, a_{s}\right)$ is generated by $\left\{m \in \mathcal{B}\left(a_{1}, \ldots, a_{s}\right):|m| \leq \delta_{0}(H)\right\}$. If $a_{1}, \ldots, a_{s}$ are distinct, then we are done by definition of $\delta_{0}$. Otherwise suppose $a_{1}=a_{2}$. Clearly $\delta_{0}(H) \geq \operatorname{ord}_{H}\left(a_{1}\right)=$ $q$, and $n:=(1, q-1,0, \ldots, 0) \in \mathcal{B}\left(a_{1}, \ldots, a_{s}\right)$ satisfies $|n| \leq \delta_{0}(H)$. Moreover, given any $m \in \mathcal{G}\left(a_{1}, \ldots, a_{s}\right)$, replace $m$ by $\tilde{m}:=m-m_{1} n$. Then $\tilde{m}$ belongs to $\mathcal{G}\left(a_{2}, \ldots, a_{s}\right)$ (viewed as the subset of $\mathcal{G}\left(a_{1}, \ldots, a_{s}\right)$ consisting of the elements whose first coordinate is zero). By the induction hypothesis $\tilde{m}$ belongs to the group generated by $\left\{m \in \mathcal{B}\left(a_{2}, \ldots, a_{s}\right):|m| \leq \delta_{0}(H)\right\}$, implying in turn that $m$ belongs to the group generated by $\left\{m \in \mathcal{B}\left(a_{1}, \ldots, a_{s}\right):|m| \leq \delta_{0}(H)\right\}$. This shows $\delta(H)=\delta_{0}(H)$.

Now take a $G$-module $V$ with the notation of the beginning of Section 2, For any subset $\left\{i_{1}<\cdots<i_{s}\right\} \subset\{1, \ldots, k\}$ with $s \leq \kappa(\widehat{G})$ the abelian group $\mathcal{G}\left(\chi_{i_{1}}, \ldots, \chi_{i_{s}}\right)$ is generated by the elements $m \in \mathcal{B}\left(\chi_{i_{1}}, \ldots, \chi_{i_{s}}\right)$ with $|m| \leq \delta(\widehat{G})$. It follows by Theorem 2.1 that $\left\{x^{m}: m \in \mathcal{B}\left(\chi_{1}, \ldots, \chi_{k}\right), \quad|m| \leq \delta(\widehat{G})\right\}$ is a separating set in $\mathcal{O}(V)^{G}$. Thus $\beta_{\text {sep }}(G, V) \leq \delta(\widehat{G})$. Since $V$ was an arbitrary $G$-module, we deduce the inequality $\beta_{\text {sep }}(G) \leq \delta(\widehat{G})$. Note finally that the isomorphism $G \cong \widehat{G}$ implies $\delta(G)=\delta(\widehat{G})$. Combining with the first paragraph we obtain $\beta_{\text {sep }}(G) \leq \delta_{0}(G)$.

To show the reverse inequality $\beta_{\operatorname{sep}}(G) \geq \delta_{0}(G)$, take a sequence $\chi_{1}, \ldots, \chi_{k}$ of characters of $G$ such that $k \leq \kappa(\widehat{G})$ and the abelian group $\mathcal{G}\left(\chi_{1}, \ldots, \chi_{k}\right)$ is not generated by $\left\{m \in \mathcal{B}\left(\chi_{1}, \ldots, \chi_{k}\right):|m|<\delta_{0}(\widehat{G})\right\}$. Such a sequence $\chi_{1}, \ldots, \chi_{k}$ exists by definition of $\delta_{0}(\widehat{G})$. It follows by Theorem 2.1 that $\left\{x^{m}: m \in \mathcal{B}\left(\chi_{1}, \ldots, \chi_{k}\right), \quad|m|<\right.$ $\left.\delta_{0}(G)\right\}$ is not a separating set in $\mathbb{F}\left[x_{1}, \ldots, x_{k}\right]^{G}=\mathcal{O}(V)^{G}$, where the $G$-module $V$ is determined by $g \cdot x_{i}=\chi_{i}(g) x_{i}$ for $i=1, \ldots, k$. Taking into account (11) we conclude $\beta_{\text {sep }}(G, V) \geq \delta_{0}(G)$, implying in turn $\beta_{\text {sep }}(G) \geq \delta_{0}(G)$.

\section{Degree bounds}

We fix the following notation for the whole Section. Decompose our (additively written) abelian group $G$ as a direct product of cyclic groups

$$
G=C_{n_{1}} \oplus \cdots \oplus C_{n_{r}}
$$

where $n_{r}\left|n_{r-1}\right| \cdots \mid n_{1}$ and $n_{r}>1$, so in particular $n_{1}$ is the exponent of $G$ and $r$ is the rank (the minimal number of generators) of $G$, hence the Helly dimension of $G$ is $\kappa(G)=r+1$. Set

$$
\mathrm{d}^{*}(G)=\sum_{i=1}^{r}\left(n_{i}-1\right) .
$$

It is well known that

$$
\mathrm{d}^{*}(G)+1 \leq \mathrm{D}(G)
$$

where $\mathrm{D}(G)$ is the Davenport constant of $G$ (cf. Section 11). Classical results in arithmetic combinatorics assert that we have equality in (3) if $G$ is a $p$-group or $G$ has rank two. On the other hand there are some infinite sequences of finite abelian groups for which the inequality in (3) is known to be strict. Beyond that it is not well understood when equality holds in (3). We refer to the surveys [14] and [13] for the above results and for references on zero-sum sequences in finite abelian groups.

We shall need the following technical and elementary lemma. 
Lemma 3.1. Let $\left(n_{1}, \ldots, n_{r}\right),\left(m_{1}, \ldots, m_{r}\right) \in \mathbb{N}^{r}$ be $r$-tuples of positive integers such that the following divisibility conditions hold for them:

$$
m_{i} \mid n_{i} \quad \text { for } \quad i=1, \ldots, r, \quad n_{i+1} \mid m_{i} \quad \text { for } \quad i=1, \ldots, r-1 .
$$

Then the following inequality holds:

$$
\sum_{i=1}^{r}\left(n_{i}-1\right) \geq \sum_{i=1}^{r}\left(m_{i}-1\right)+\prod_{i=1}^{r} \frac{n_{i}}{m_{i}}-1 .
$$

Moreover, equality holds in (4) if and only if there exists a $j \in\{1,2, \ldots, r\}$ such that $m_{1}=n_{1}, \ldots, m_{j}=n_{j}, m_{j+1}=n_{j+2}, m_{j+2}=n_{j+3}, \ldots$ (where we mean that $\left.n_{r+1}=1\right)$.

Proof. When $r=1$, (4) becomes $n_{1}-1 \geq\left(m_{1}-1\right)+\frac{n_{1}}{m_{1}}-1$, which is equivalent to the obvious $\left(m_{1}-1\right)\left(\frac{n_{1}}{m_{1}}-1\right) \geq 0$. Assume from now on that $r>1$. If $n_{1}=m_{1}$, then we may omit them and deal with the sequences $\left(n_{2}, \ldots, n_{r}\right)$ and $\left(m_{2}, \ldots, m_{r}\right)$, since the inequality (4) for these shorter sequences is obviously equivalent to the corresponding inequality for the original sequences. So from now on we assume that $n_{1}>m_{1}$, that is, $m_{1}$ is a proper divisor of $n_{1}$.

The conditions imply that $\prod_{i=1}^{r} \frac{n_{i}}{m_{i}}$ divides $n_{1}$, and $\prod_{i=1}^{r} \frac{n_{i}}{m_{i}}=n_{1}$ if and only if $m_{r}=1, n_{r}=m_{r-1}, \ldots, n_{2}=m_{1}$. Assume first that these equalities hold. Then we have

$$
\sum_{i=1}^{r}\left(n_{i}-1\right)=\prod_{i=1}^{r} \frac{n_{i}}{m_{i}}-1+\sum_{i=2}^{r}\left(n_{i}-1\right)=\prod_{i=1}^{r} \frac{n_{i}}{m_{i}}-1+\sum_{i=1}^{r-1}\left(m_{i}-1\right)
$$

and taking into account that $m_{r}=1$, we see that (4) holds with equality in this case. Suppose finally that $\prod_{i=1}^{r} \frac{n_{i}}{m_{i}}$ is a proper divisor of $n_{1}$. Let $p$ be a minimal prime divisor of $n_{1}$. Then $\prod_{i=1}^{r} \frac{n_{i}}{m_{i}} \leq \frac{n_{1}}{p}$ and $m_{1} \leq \frac{n_{1}}{p}$. Consequently we have

$$
n_{1}-1=\frac{n_{1}}{p} \cdot p-1 \geq \frac{n_{1}}{p}+\frac{n_{1}}{p}-1 \geq \prod_{i=1}^{r} \frac{n_{i}}{m_{i}}+m_{1}-1>\prod_{i=1}^{r} \frac{n_{i}}{m_{i}}-1+\left(m_{1}-1\right) .
$$

Since for $i=2, \ldots r$ we have $n_{i}-1 \geq m_{i}-1$, we conclude (44).

Lemma 4.1 in [17. (see also Exercise 1.6 in [16]) asserts that $\mathrm{d}^{*}(G) \geq \mathrm{d}^{*}(H)+$ $\mathrm{d}^{*}(G / H)$ for any subgroup $H$ of $G$. In Lemma 3.2 we provide a detailed proof of the special case when $G / H$ is cyclic, yielding also a characterization of the case when equality holds.

Lemma 3.2. Let $H$ be a proper subgroup of $G$ such that the factor group $G / H$ is cyclic. Then $\mathrm{d}^{*}(G) \geq \mathrm{d}^{*}(H)+[G: H]-1$, with equality only if $\operatorname{rank}(H)=$ $\operatorname{rank}(G)-1$, and $H \cong \bigoplus_{i \in\{1, \ldots, r\} \backslash\{j\}} C_{n_{i}}$ for some $j \in\{1, \ldots, r\}$.

Proof. Take a finite abelian $p$-group $A$. It is isomorphic to $C_{p^{\lambda_{1}}} \oplus \cdots \oplus C_{p^{\lambda_{k}}}$ where $\lambda_{1} \geq \cdots \geq \lambda_{k}>0$. We call the partition $\lambda=\left(\lambda_{1}, \ldots, \lambda_{k}\right)$ the type of $A$. Any subgroup $B$ of $A$ has type $\mu=\left(\mu_{1}, \ldots, \mu_{k}\right), \mu_{1} \geq \cdots \geq \mu_{k} \geq 0$, where $\mu_{i} \leq \lambda_{i}$ for $i=1, \ldots, k$. Moreover, $A$ has a subgroup $B$ of type $\mu$ such that the factor group $A / B$ is cyclic (necessarily of order $p^{d}$, where $d:=\sum\left(\lambda_{i}-\mu_{i}\right)$ ) if and only if the Littlewood-Richardson coefficient $c_{\mu,(d)}^{\lambda}$ is non-zero (see for example II.4.3 in [21]), and by Pieri's rules (see for example I.5.16 in [21] ) this happens if and only if the additional inequalities $\mu_{1} \geq \lambda_{2}, \mu_{2} \geq \lambda_{3}, \ldots, \mu_{r-1} \geq \lambda_{r}$ hold as well. 
Note that both $G$ and $H$ are the direct products of their unique Sylow subgroups. Therefore it follows from the above paragraph that the subgroup $H$ is isomorphic to $H \cong C_{m_{1}} \oplus \cdots \oplus C_{m_{r}}$ where $1 \leq m_{r}\left|m_{r-1}\right| \cdots \mid m_{1}$, and $m_{i} \mid n_{i}$ for $i=1, \ldots, r$. Moreover, since $G / H$ is cyclic, for any prime $p$ the factor of the Sylow $p$-subgroup of $G$ modulo the Sylow $p$-subgroup of $H$ is cyclic, so again by the above paragraph the conditions $n_{i+1} \mid m_{i}$ for $i=1, \ldots, r-1$ hold as well. This means that the assumptions of Lemma 3.1 hold for the $r$-tuples $\left(n_{1}, \ldots, n_{r}\right),\left(m_{1}, \ldots, m_{r}\right)$. Thus by Lemma 3.1 the inequality (4) holds, which is the same as the desired inequality in our statement.

Given the finite abelian groups $G, H, K$, there exists a subgroup $G_{1}$ of $G$ such that $G_{1} \cong H$ and $G / G_{1} \cong K$ if and only if there exists a subgroup $G_{2}$ of $G$ with $G_{2} \cong K$ and $G / G_{2} \cong H$. Therefore Lemma 3.2 has its dual form as well:

Lemma 3.3. Let $K$ be a nontrivial cyclic subgroup of $G$. Then $\mathrm{d}^{*}(G) \geq \mathrm{d}^{*}(G / K)+$ $|K|-1$, with equality only if $\operatorname{rank}(G / K)=\operatorname{rank}(G)-1$, and for some $j \in\{1, \ldots, r\}$ we have $G / K \cong \bigoplus_{i \in\{1, \ldots, r\} \backslash\{j\}} C_{n_{i}}$.

Corollary 3.4. Let $a_{1}, \ldots, a_{k}$ be a sequence of elements generating $G$, and for $i=1, \ldots, k$ denote by $d_{i}$ the order of $a_{i}$ modulo the subgroup $\left\langle a_{1}, \ldots, a_{i-1}\right\rangle$ (so $\left.d_{1} \ldots d_{k}=|G|\right)$. Then $\mathrm{d}^{*}(G) \geq \sum_{i=1}^{k}\left(d_{i}-1\right)$, with equality only if the multiset $\left\{n_{1}, \ldots, n_{r}\right\}$ coincides with the multiset $\left\{d_{i_{1}}, \ldots, d_{i_{r}}\right\}$ obtained by omitting all occurrances of 1 in the sequence $d_{1}, \ldots, d_{k}$.

Proof. Apply induction for $k$. The case $k=1$ is obvious, since then $G$ is cyclic of order $d_{1}$, so $r=1, n_{1}=d_{1}$, and $\mathrm{d}^{*}(G)=d_{1}-1=n_{1}-1$. Assume next that $k>1$, and set $H:=\left\langle a_{1}, \ldots, a_{k-1}\right\rangle$. If $H=G$, then $d_{k}=1, \mathrm{~d}^{*}(G)=\mathrm{d}^{*}(H)$, and the statement follows by the induction hypothesis applied to $H$. If $H$ is a proper subgroup of $G$, then $d_{k}>1$. By Lemma 3.2 we have $\mathrm{d}^{*}(G) \geq \mathrm{d}^{*}(H)+d_{k}-1$, with equality only if $H \cong \bigoplus_{i \in\{1, \ldots, r\} \backslash\{j\}} C_{n_{i}}$ for some $j \in\{1, \ldots, r\}$, implying also $n_{j}=d_{k}$. Now we may conclude by applying the induction hypothesis for $H$ and the sequence $a_{1}, \ldots, a_{k-1}$.

We shall use the following terminology. Given an ordered sequence $a_{1}, \ldots, a_{k} \in$ $G$ of elements generating $G$, any $b \in G$ can be uniquely written as

$$
b=\sum_{i=1}^{k} l_{i} a_{i}, \quad 0 \leq l_{i} \leq d_{i}-1 \text { for } i=1, \ldots, k
$$

where $d_{i}$ denotes the smallest positive integer $d$ such that $d a_{i}$ belongs to the subgroup $\left\langle a_{1}, \ldots, a_{i-1}\right\rangle$. Indeed, consider the chain

$$
\{0\} \subset\left\langle a_{1}\right\rangle \subset\left\langle a_{1}, a_{2}\right\rangle \subset \cdots \subset\left\langle a_{1}, \ldots, a_{k}\right\rangle=G
$$

of subgroups. It has cyclic factors of order $d_{1}, \ldots, d_{k}$. The factor $G /\left\langle a_{1}, \ldots, a_{k-1}\right\rangle$ is generated by the coset of $a_{k}$, hence $b+\left\langle a_{1}, \ldots, a_{k-1}\right\rangle=l\left(a_{k}+\left\langle a_{1}, \ldots, a_{k-1}\right\rangle\right)$ for a unique $0 \leq l \leq d_{k}-1$. Now continue in the same way with the element $b-l a_{k}$ in the group $\left\langle a_{1}, \ldots, a_{k-1}\right\rangle$. We shall refer to (15) as the normal form of $b$ with respect to $a_{1}, \ldots, a_{k}$, and we call $\sum_{i=1}^{k}\left(d_{i}-1-l_{i}\right)$ the deficit of $b$.

Lemma 3.5. Let $a_{1}, \ldots, a_{k}$ be an arbitrary sequence of elements in $G$, and denote by $d_{k}$ the order of $a_{k}$ modulo the subgroup $\left\langle a_{1}, \ldots, a_{k-1}\right\rangle$. Then there exists an $m=\left(m_{1}, \ldots, m_{k}\right) \in \mathcal{B}\left(a_{1}, \ldots, a_{k}\right)$ such that $m_{k}=d_{k}$ and $|m|=m_{1}+\cdots+m_{k} \leq$ $\mathrm{d}^{*}(G)+1$. 
Proof. The group $\left\langle a_{1}, \ldots, a_{k-1}\right\rangle$ contains $d_{k} a_{k}$. Set $m:=\left(l_{1}, \ldots, l_{k-1}, d_{k}\right)$ where $-d_{k} a_{k}=\sum_{i=1}^{k-1} l_{i} a_{i}$ is the normal form of $-d_{k} a_{k}$ with respect to $a_{1}, \ldots, a_{k-1}$. Then $m$ belongs to $\mathcal{B}\left(a_{1}, \ldots, a_{k}\right)$, and $|m| \leq \sum_{i=1}^{k-1}\left(d_{i}-1\right)+d_{k} \leq \mathrm{d}^{*}(G)+1$, where the last inequality holds by Corollary 3.4

Lemma 3.6. Suppose that $a_{1}, \ldots, a_{k}$ are distinct non-zero elements in $G$, and denote by $d_{i}$ the order of $a_{i}$ modulo $\left\langle a_{1}, \ldots, a_{i-1}\right\rangle$ for $i=1, \ldots, k$. If there is no $m \in \mathcal{B}\left(a_{1}, \ldots, a_{k}\right)$ such that $|m| \leq \mathrm{d}^{*}(G)$ and $m_{k}=d_{k}$, then either $k=1$ and $G=\left\langle a_{1}\right\rangle$, or $G=\left\langle a_{1}, \ldots, a_{k-1}\right\rangle$, the multiset $\left\{d_{1}, \ldots, d_{k-1}\right\}$ coincides with the multiset $\left\{n_{1}, \ldots, n_{r}\right\}$ (so in particular $k-1=r$ is the rank of $G$ ), and the deficit of $-a_{k}$ with respect to $a_{1}, \ldots, a_{k-1}$ is zero.

Proof. Suppose that $k>1$ and for the $m$ constructed in the proof of Lemma 3.5 we have $|m|=\mathrm{d}^{*}(G)+1$, so

$$
-d_{k} a_{k}=\sum_{i=1}^{k-1}\left(d_{i}-1\right) a_{i}
$$

and $\mathrm{d}^{*}(G)=\sum_{j=1}^{k}\left(d_{j}-1\right)$. Assume first that for some $i \in\{1, \ldots, k-1\}$ we have $d_{i}=1$. Then $a_{i} \in\left\langle a_{1}, \ldots, a_{i-1}\right\rangle$, so we may write

$$
a_{i}=l_{1} a_{1}+\cdots+l_{i-1} a_{i-1}
$$

in its normal form with respect to $a_{1}, \ldots, a_{i-1}$. Equations (6) and (7) imply

$$
-d_{k} a_{k}=\sum_{j=1}^{i-1}\left(d_{j}-1-l_{j}\right)+a_{i}+\sum_{q=i+1}^{k-1}\left(d_{q}-1\right) a_{q} .
$$

Setting $m^{\prime}:=\left(d_{1}-1-l_{1}, \ldots, d_{i-1}-1-l_{i-1}, 1, d_{i+1}-1, \ldots, d_{k-1}-1, d_{k}\right)$ we get that $m^{\prime} \in \mathcal{B}\left(a_{1}, \ldots a_{k}\right)$. Moreover, as $a_{1}, \ldots, a_{i}$ are distinct, we have that $l_{1}+\cdots+l_{i-1} \geq$ 2 , hence $\left|m^{\prime}\right|=\sum_{i=1}^{k}\left(d_{i}-1\right)+2-\left(l_{1}+\cdots+l_{i-1}\right) \leq \sum_{j=1}^{k}\left(d_{j}-1\right)=\mathrm{d}^{*}(G)$. So we found an $m^{\prime} \in \mathcal{B}\left(a_{1}, \ldots, a_{k}\right)$ with $m_{k}^{\prime}=d_{k}$ and $\left|m^{\prime}\right| \leq \mathrm{d}^{*}(G)$.

It remains to deal with the case when $d_{1}, \ldots, d_{k-1}$ are all greater than 1. Suppose first that $H:=\left\langle a_{1}, \ldots, a_{k-1}\right\rangle \subsetneq G$. If $d_{k}=1$, then $\left\langle a_{1}, \ldots, a_{k}\right\rangle=H \subsetneq G$, and by Lemma 3.5 there exists an $m \in \mathcal{B}\left(a_{1}, \ldots, a_{k}\right)$ with $m_{k}=d_{k}$ and $|m| \leq \mathrm{d}^{*}(H)+$ $1 \leq \mathrm{d}^{*}(G)$. If $d_{i}>1$ for all $i=1, \ldots, k$, by Corollary 3.4 the equality $\mathrm{d}^{*}(G)=$ $\sum_{j=1}^{\bar{k}}\left(d_{j}-1\right)$ implies that the multiset $\left\{d_{1}, \ldots, d_{k}\right\}$ coincides with $\left\{n_{1}, \ldots, n_{r}\right\}$. In particular, $k=r$ is the rank of $G$. However, since $d_{1}$ is the order of $a_{1}$, equation (6) implies that $a_{1}$ is contained in $\left\langle a_{2}, \ldots, a_{k}\right\rangle$. Thus $G$ can be generated by $k-1=$ $r-1$ elements. This is a contradiction, so this case does not occur. Finally, if $\left\langle a_{1}, \ldots, a_{k-1}\right\rangle=G$, then $d_{k}=1$, and (6) becomes $-a_{k}=\sum_{i=1}^{k-1}\left(d_{i}-1\right) a_{i}$, so the deficit of $-a_{k}$ is zero. Moreover, the equality $\mathrm{d}^{*}(G)=\sum_{j=1}^{k}\left(d_{j}-1\right)=\sum_{j=1}^{k-1}\left(d_{j}-1\right)$ implies by Corollary 3.4 that the multisets $\left\{d_{1}, \ldots, d_{k-1}\right\}$ and $\left\{n_{1}, \ldots, n_{r}\right\}$ coincide, finishing the proof.

Lemma 3.7. Let $a_{1}, \ldots, a_{k}$ be a sequence of elements of a non-cyclic group $G$, and denote by $g_{i}$ the order of $a_{i}$ modulo the subgroup $\left\langle a_{1}, \ldots, a_{i-1}, a_{i+1}, \ldots, a_{k}\right\rangle$. Assume that the following hold:

(a) There does not exist an $m=\left(m_{1}, \ldots, m_{k}\right) \in \mathcal{B}\left(a_{1}, \ldots, a_{k}\right)$ with $|m| \leq$ $\mathrm{d}^{*}(G)$ such that $m_{i}=g_{i}$ for some $i \in\{1, \ldots, k\}$. 
(b) There does not exist a pair $m^{\prime}, m^{\prime \prime} \in \mathcal{B}\left(a_{1}, \ldots, a_{k}\right)$ with $\left|m^{\prime}\right| \leq \mathrm{d}^{*}(G)$, $\left|m^{\prime \prime}\right| \leq \mathrm{d}^{*}(G)$, such that $m_{i}^{\prime}=2$ and $m_{i}^{\prime \prime}=3$ for some $i \in\{1, \ldots, k\}$.

Then $2=n_{s+1}=\cdots=n_{r}$ where $r=2 s$ or $r=2 s-1$.

Proof. No element in $\left\{a_{1}, \ldots, a_{k}\right\}$ is zero, since $a_{i}=0$ implies $g_{i}=1$, and $e_{i} \in$ $\mathcal{B}\left(a_{1}, \ldots, a_{k}\right)$ where $e_{i}$ is the $i$ th standard basis vector in $\mathbb{Z}^{k}$, hence (a) implies $\mathrm{d}^{*}(G)<1$, a contradiction. The elements $a_{1}, \ldots, a_{k}$ are distinct. Indeed, assume to the contrary that say $a_{1}=a_{2}$. Then denoting by $d$ the order of $a_{1}$, we have that $g_{1}=1$ and $m=(1, d-1,0, \ldots, 0) \in \mathcal{B}\left(a_{1}, \ldots, a_{k}\right)$, hence by (a) we have $\mathrm{d}^{*}(G)<d$, a contradiction (recall that $G$ is not cyclic).

Thus $a_{1}, \ldots, a_{k}$ are distinct non-zero elements of $G$, and Lemma 3.6 applies for them with an arbitrary ordering of the elements in the sequence. In particular, by condition (a) and Lemma 3.6 the rank of $G$ is $k-1$, and any $k-1$ of the elements $a_{1}, \ldots, a_{k}$ generate $G$. Furthermore, after an arbitrary renumbering of the elements in the set $\left\{a_{1}, \ldots, a_{k}\right\}$, the deficit of $-a_{k}$ with respect to $a_{1}, \ldots, a_{k-1}$ is 0 , and the multiset $\left\{d_{1}, \ldots, d_{k-1}\right\}$ coincides with $\left\{n_{1}, \ldots, n_{r}\right\}$, where $d_{i}$ stands for the order of $a_{i}$ modulo $\left\langle a_{1}, \ldots, a_{i-1}\right\rangle$.

We claim that for any $i \in\{1, \ldots, k\}$ with $2 a_{i} \neq 0$ there exists a $j \neq i$ such that $2 a_{i}=2 a_{j}$. Indeed, suppose for example that $2 a_{k} \neq 0$. Recall that the deficit of $-a_{k}$ with respect to $a_{1}, \ldots, a_{k-1}$ is zero, so

$$
-a_{k}=\sum_{i=1}^{k-1}\left(d_{i}-1\right) a_{i} .
$$

Since $-a_{k} \neq-2 a_{k}$, the deficit of $-2 a_{k}$ is different from the deficit of $-a_{k}$, so the deficit of $-2 a_{k}$ is non-zero. Also $a_{k}$ is different from each of $a_{1}, \ldots, a_{k-1}$, implying that the deficit of $-2 a_{k}$ is not 1 . Consequently, the deficit of $-2 a_{k}$ is at least 2 , hence $m^{\prime}:=\left(l_{1}, \ldots, l_{k-1}, 2\right)$ where $-2 a_{k}=l_{1} a_{1}+\cdots+l_{k-1} a_{k-1}$ is the normal form of $-2 a_{k}$ satisfies $m_{k}^{\prime}=2$ and $\left|m^{\prime}\right|=2+l_{1}+\cdots+l_{k-1} \leq \mathrm{d}^{*}(G)$. It follows by assumption (b) that the deficit of $-3 a_{k}$ is at most 2 . It can not be 0 , the deficit of $-a_{k}$, since $2 a_{k} \neq 0$, and it can not be 1 , otherwise $2 a_{k}$ coincides with one of $a_{1}, \ldots, a_{k-1}$, say $2 a_{k}=a_{2}$, and therefore $G=\left\langle a_{2}, a_{3}, \ldots, a_{k}\right\rangle=\left\langle a_{3}, \ldots, a_{k}\right\rangle$ is generated by $k-2=r-1$ elements, a contradiction. Thus the deficit of $-3 a_{k}$ is 2. There are two possible cases: $-3 a_{k}=\left(d_{1}-2\right) a_{1}+\left(d_{2}-2\right) a_{2}+\sum_{i=3}^{k-1}\left(d_{i}-1\right) a_{i}$ or $-3 a_{3}=\left(d_{1}-3\right) a_{1}+\left(d_{2}-1\right) a_{2}+\sum_{i=3}^{k-1}\left(d_{i}-1\right) a_{i}$ (with a suitable ordering of $\left.a_{1}, \ldots, a_{k-1}\right)$. Comparing this with (8) in the first case we deduce $2 a_{k}=a_{1}+a_{2}$, hence $-a_{1}=-2 a_{k}+a_{2}$. The latter equality shows that the deficit of $-a_{1}$ with respect to $a_{k}, a_{2}, a_{3}, \ldots, a_{k-1}$ can not be zero, a contradiction. Thus this case does not occur. The only remaining possibility is that $-3 a_{k}=\left(d_{1}-3\right) a_{1}+\sum_{i=2}^{k-1}\left(d_{i}-1\right) a_{i}$ (with a suitable ordering of $a_{1}, \ldots, a_{k-1}$ ). Comparing this with (8) we conclude $2 a_{k}=2 a_{1}$. So the claim is proved.

It follows from the above claim that the set $\left\{2 a_{1}, \ldots, 2 a_{k}\right\}$ contains at most $\frac{k}{2}$ non-zero elements, hence the rank of the group $\left\langle 2 a_{1}, \ldots, 2 a_{k}\right\rangle=\{2 a: a \in G\}$ is at most $\frac{r+1}{2}$. On the other hand the rank of $\{2 a: a \in G\}$ equals $\mid\left\{i \in\{1, \ldots, r\}: n_{i}>\right.$ $2\} \mid$. Consequently we have $\left|\left\{j \in\{1, \ldots, r\}: n_{j}=2\right\}\right| \geq \frac{r-1}{2}$.

Proposition 3.8. Suppose that $G=C_{n_{1}} \oplus \cdots \oplus C_{n_{s}} \oplus C_{2} \oplus \cdots \oplus C_{2}$ where $r=2 s-1$ or $r=2 s$, so $2=n_{s+1}=\cdots=n_{r}$, and $2\left|n_{s}\right| n_{s-1}|\cdots| n_{1}$. Denote by $e_{1}, \ldots, e_{s}, f_{1}, \ldots, f_{r-s}$ the generators of the direct factors of $G$, thus the order of $e_{i}$ is $n_{i}$ for $i=1, \ldots, s$, and the order of $f_{j}$ is 2 for $j=1, \ldots, r-s$. Set $a_{1}=e_{1}$, 
$a_{2 i}=e_{i}+f_{i}$ and $a_{2 i+1}=f_{i}+e_{i+1}$ for $i=1, \ldots, s-1$, and $a_{2 s}=e_{s}$ if $r=2 s-1$ whereas $a_{2 s}=e_{s}+f_{s}, a_{2 s+1}=f_{s}$ if $r=2 s$. Then the abelian group $\mathcal{G}\left(a_{1}, \ldots, a_{r+1}\right)$ is not generated by $\left\{m \in \mathcal{B}\left(a_{1}, \ldots, a_{r+1}\right):|m| \leq \mathrm{d}^{*}(G)\right\}$.

Proof. The element $a_{1}$ is contained in the group $\left\langle a_{2}, \ldots, a_{r+1}\right\rangle$, whence there exists an element $u \in \mathcal{B}\left(a_{1}, \ldots, a_{r+1}\right)$ with $u_{1}=1$. On the other hand we shall show that for any $m \in \mathcal{B}\left(a_{1}, \ldots, a_{r+1}\right)$ with $|m| \leq \mathrm{d}^{*}(G)$ we have that $m_{1}$ is even, and consequently $u$ is not contained in the subgroup of $\mathcal{G}\left(a_{1}, \ldots, a_{r+1}\right)$ generated by $\left\{m \in \mathcal{B}\left(a_{1}, \ldots, a_{r+1}\right):|m| \leq \mathrm{d}^{*}(G)\right\}$. Indeed, take $m=\left(m_{1}, \ldots, m_{r+1}\right) \in$ $\mathcal{B}\left(a_{1}, \ldots, a_{r+1}\right)$ with $m_{1}$ odd and $|m|$ minimal possible. Consider the case when $r=2 s-1$, and so $\mathrm{d}^{*}(G)=n_{1}+\cdots+n_{s}-1$. The order of $a_{2 i-1}$ and $a_{2 i}$ is $n_{i}$, hence $0 \leq m_{2 i}, m_{2 i-1} \leq n_{i}-1$ hold for $i=1, \ldots, s$. We have

$$
0=\sum_{i=1}^{2 s} m_{i} a_{i}=\sum_{i=1}^{s}\left(m_{2 i-1}+m_{2 i}\right) e_{i}+\sum_{i=1}^{s-1}\left(m_{2 i}+m_{2 i+1}\right) f_{i} .
$$

From the coefficient of $e_{1}$ above we deduce that $m_{1}+m_{2}=n_{1}$, hence $m_{2}$ is odd. From the coefficient of $f_{1}$ above we infer that $m_{3}$ is odd as well. From the coefficient of $e_{2}$ above we deduce that $m_{3}+m_{4}=n_{2}$, and consequently $m_{4}$ is odd. Continuing in the same way and looking at step-by-step the coefficient of $f_{2}, e_{3}, f_{3}, e_{4}, \ldots, f_{s-1}, e_{s}$ we arrive at the conclusion that $m_{2 i-1}+m_{2 i}=n_{i}$ for all $i=1, \ldots, s$, whence $|m|=\sum_{i=1}^{s}\left(m_{2 i-1}+m_{2 i}\right)=\sum_{i=1}^{s} n_{i}>\left(\sum_{i=1}^{s} n_{i}\right)-1=\mathrm{d}^{*}(G)$. The case when $r=2 s$ is similar.

Proposition 3.9. Let $a_{1}, \ldots, a_{k}$ be a sequence of elements of $G$.

(i) The abelian group $\mathcal{G}\left(a_{1}, \ldots, a_{k}\right)$ is generated by $\left\{m \in \mathcal{B}\left(a_{1}, \ldots, a_{k}\right):|m| \leq\right.$ $\left.\mathrm{d}^{*}(G)+1\right\}$.

(ii) If $r>1$ and $n_{s+1} \neq 2$ where $r=2 s$ or $r=2 s-1$, then $\mathcal{G}\left(a_{1}, \ldots, a_{k}\right)$ is generated by $\left\{m \in \mathcal{B}\left(a_{1}, \ldots, a_{k}\right):|m| \leq \mathrm{d}^{*}(G)\right\}$.

Proof. (i) Take an arbitrary $u \in \mathcal{G}\left(a_{1}, \ldots, a_{k}\right)$. Since $u_{k} a_{k}=-\sum_{i=1}^{k-1} u_{i} a_{i}$ belongs $\left\langle a_{1}, \ldots, a_{k-1}\right\rangle$, there exists an integer $l_{1}$ such that $u_{k}=l_{1} d_{k}$, where $d_{k}$ is the order $a_{k}$ modulo $\left\langle a_{1}, \ldots, a_{k-1}\right\rangle$. By Lemma 3.5 there exists an $m^{(1)} \in \mathcal{B}\left(a_{1}, \ldots, a_{k}\right)$ with $m_{k}^{(1)}=d_{k}$ and $\left|m^{(1)}\right| \leq \mathrm{d}^{*}(G)+1$. Set $u^{\prime}:=u-l_{1} m^{(1)}$. Then $u_{k}^{\prime}=0$, so $u^{\prime}$ belongs to $\mathcal{G}\left(a_{1}, \ldots, a_{k-1}\right)$ identified with the subset $\left\{m \in \mathcal{G}\left(a_{1}, \ldots, a_{k}\right): m_{k}=\right.$ $0\}$ in $\mathcal{G}\left(a_{1}, \ldots, a_{k}\right)$. Repeat the same step for $u^{\prime}$ to obtain $l_{2} \in \mathbb{Z}$ and $m^{(2)} \in$ $\mathcal{B}\left(a_{1}, \ldots, a_{k-1}\right)$ such that $\left|m^{(2)}\right| \leq \mathrm{d}^{*}(G)+1$ and $u^{\prime}-l_{2} m^{(2)} \in \mathcal{G}\left(a_{1}, \ldots, a_{k-2}\right)$. Continue in the same way, eventually we get that

$$
u=\sum_{i=1}^{k} l_{i} m^{(i)} \text { where } m^{(i)} \in \mathcal{B}\left(a_{1}, \ldots, a_{i}\right), \quad\left|m^{(i)}\right| \leq \mathrm{d}^{*}(G)+1 \text { for } i \in\{1, \ldots, k\} .
$$

(ii) We slightly adjust the poof of (i). By our assumption on $G$, it follows from Lemma 3.7 that after a possible reordering of the elements $a_{1}, \ldots, a_{k}$ and denoting by $g_{k}$ the order of $a_{k}$ modulo $\left\langle a_{1}, \ldots, a_{k-1}\right\rangle$ at least one of the following two possibilities holds:

(a) there is an $m \in \mathcal{B}\left(a_{1}, \ldots, a_{k}\right)$ with $|m| \leq \mathrm{d}^{*}(G)$ and $m_{k}=g_{k}$;

(b) there are $m^{\prime}, m^{\prime \prime} \in \mathcal{B}\left(a_{1}, \ldots, a_{k}\right)$ with $\left|m^{\prime}\right|,\left|m^{\prime \prime}\right| \leq \mathrm{d}^{*}(G)$ and $m_{k}^{\prime}=2$, $m_{k}^{\prime \prime}=3$.

Now take an arbitrary $u \in \mathcal{G}\left(a_{1}, \ldots, a_{k}\right)$. We have $u_{k}=l g_{k}$ for some $l \in \mathbb{Z}$. Set $u^{\prime}:=u-l m$ if (a) holds and $u^{\prime}:=u-l\left(m^{\prime \prime}-m^{\prime}\right)$ if (b) holds (note that 
in this case necessaily $\left.g_{k}=1\right)$. Then $u^{\prime}$ belongs to $\mathcal{G}\left(a_{1}, \ldots, a_{k-1}\right)$. Continue in the same way with the sequence $a_{1}, \ldots, a_{k-1}$ and $u^{\prime} \in \mathcal{G}\left(a_{1}, \ldots, a_{k-1}\right)$. In $k$ steps we get a presentation of $u$ as an integral linear combination of elements from $\left\{m \in \mathcal{B}\left(a_{1}, \ldots, a_{k}\right):|m| \leq \mathrm{d}^{*}(G)\right\}$.

Theorem 3.10. For any finite abelian group $G$ we have the inequality

$$
\beta_{\text {sep }}(G) \leq \mathrm{d}^{*}(G)+1
$$

with equality holding if and only if $G$ is cyclic or $2=n_{s+1}=\cdots=n_{r}$ where $r=2 s-1$ or $r=2 s$.

Proof. Proposition 3.9 (i) and Corollary 2.6 imply the inequality $\beta_{\text {sep }}(G) \leq \mathrm{d}^{*}(G)+$ 1. Furthermore, if $G$ is not cyclic and $n_{s+1} \neq 2$ where $r=2 s$ or $r=2 s-1$, then by Proposition 3.9 (ii) and Corollary 2.6 we even get the stronger inequality $\beta_{\text {sep }}(G) \leq \mathrm{d}^{*}(G)$.

For a cyclic group $G$ any faithful 1-dimensional $G$-module $V$ gives $\beta_{\text {sep }}(G, V)=$ $|G|=\mathrm{d}^{*}(G)+1$. Suppose finally that $2=n_{s+1}=\cdots=n_{r}$ where $r=2 s-1$ or $r=2 s$. By Proposition 3.8 and Corollary 2.6 we conclude $\beta_{\text {sep }}(G)>\mathrm{d}^{*}(G)$. Summarizing, for these groups $G$ we have the equality $\beta_{\text {sep }}(G)=\mathrm{d}^{*}(G)+1$.

Corollary 3.11. We have the strict inequality

$$
\beta_{\text {sep }}(G)<\beta(G)
$$

for any non-cyclic finite abelian group $G$ with $n_{s+1} \neq 2$, where $r=2 s-1$ or $r=2 s$.

Proof. Theorem 3.10 for a non-cyclic $G$ satisfying $n_{s+1} \neq 2$ together with (3) and (2) yields the inequalities

$$
\beta_{\text {sep }}(G) \leq \mathrm{d}^{*}(G)<\mathrm{d}^{*}(G)+1 \leq \mathrm{D}(G)=\beta(G) .
$$

Remark 3.12. Since for a finite abelian group $G$ with $n_{s+1}=\cdots=n_{r}=2$ we have $\beta_{\text {sep }}(G)=\mathrm{d}^{*}(G)+1$ by Theorem 3.10, therefore for such a group we have $\beta_{\text {sep }}(G)<$ $\beta(G)$ if and only if we have the strict inequality $\mathrm{d}^{*}(G)+1<\mathrm{D}(G)$. A complete description of the groups $G$ with $n_{s+1}=\cdots=n_{r}=2$ and $\mathrm{D}(G)>\mathrm{d}^{*}(G)+1$ is not known. On the other hand there are infinitely many known examples of groups $G$ where $n_{s+1}=\cdots=n_{r}=2$ both with equality $\mathrm{d}^{*}(G)+1=\mathrm{D}(G)$ and with strict inequality $\mathrm{d}^{*}(G)+1<\mathrm{D}(G)$, see for example Corollary 2 in [12] or Corollary 4.2.3 in [13.

\section{Acknowledgement}

I thank Alfred Geroldinger for helpful comments on the manuscript.

\section{REFERENCES}

[1] K. Cziszter, The Noether number of the non-abelian group of order $3 p$, Periodica Math. Hungarica 68 (2014), 150-159.

[2] K. Cziszter, M. Domokos, Groups with large Noether bound, Ann. Inst. Fourier (Grenoble 64 (2014), No. 3, 909-944.

[3] K. Cziszter, M. Domokos, The Noether number for the groups with a cyclic subgroup of index two, J. Algebra 399 (2014), 546-560.

[4] K. Cziszter, M. Domokos, A. Geroldinger, The interplay of invariant theory with multiplicative ideal theory and with arithmetic combinatorics, Multiplicative Ideal Theory and Factorization Theory, (Scott T. Chapman and M. Fontana and A. Geroldinger and B. Olberding, eds.), Springer-Verlag, 2016. 
[5] H. Derksen and G. Kemper, Computational Invariant Theory, volume 130 of Encyclopedia of Mathematical Sciences, Springer-Verlag, Berlin, 2002.

[6] M. Domokos, Typical separating invariants, Transform. Groups 12 (2007), 49-63.

[7] M. Domokos and E. Szabó, Helly dimension of algebraic groups, J. Lond. Math. Soc., II. Ser. 84, No. 1, 19-34 (2011).

[8] E. Dufresne and J. Jeffries Separating invariants and local cohomology, Adv. Math. 270 (2015) 565-581.

[9] B. W. Finklea, T. Moore, V. Ponomarenko, and Z. J. Turner, Invariant polynomials and minimal zero sequences, Involve 1 (2008), 159-165.

[10] P. Fleischmann, The Noether bound in invariant theory of finite groups, Adv. Math. 156 (2000), 23-32.

[11] J. Fogarty, On Noether's bound for polynomial invariants of a finite group, Electron. Res. Announc. Amer. Math. Soc. 7 (2001), 5-7.

[12] A. Geroldinger and R. Schneider, On Davenport's constant, J. Combinatorial Theory A 61(1992), 147-152.

[13] A. Geroldinger, Additive group theory and non-unique factorizations, Combinatorial Number Theory and Additive Group Theory (A. Geroldinger and I. Ruzsa, eds.), Advanced Courses in Mathematics CRM Barcelona, Birkhäuser, 2009, pp. 1-86.

[14] W. Gao and A. Geroldinger, Zero-sum problems in finite abelian groups: A survey, Expo. Math. 24 (2006), 337-369.

[15] A. Geroldinger and F. Halter-Koch, Non-Unique Factorizations. Algebraic, Combinatorial and Analytic Theory, Pure and Applied Mathematics, vol. 278, Chapman \& Hall/CRC, 2006.

[16] D. J. Grynkiewicz, Structural Additive Theory, Developments in Mathematics, Springer, 2013.

[17] D. J. Grynkiewicz, L. E. Marchan, and O. Ordaz, Representation of finite abelian group elements by subsequence sums, J. Théor. Nombres Bordx. 21 (2009), 559-587.

[18] E. Hubert and G. Labahn, Rational invariants of finite abelian groups, https://hal.inria.fr/hal-00921905v3.

[19] G. Kemper, Separating invariants, J. Symbolic Comp. 44 (2009), 1212-1222.

[20] M. Kohls and H. Kraft, Degree bounds for separating invariants, Meth. Res. Lett. 17 (2010), 1171-1182.

[21] I. G. Macdonald, Symmetric Functions and Hall Polynomials, Second Edition (1995), Clarendon Press, Oxford.

[22] M. Neusel and M. Sezer, Separating invariants for modular p-groups and groups acting diagonally, Math. Res. Lett. 16 (2009), 1029-1036.

[23] E. Noether, Der Endlichkeitssatz der Invarianten endlicher Gruppen, Math. Ann. 77 (1916), 89-92.

[24] A. Plagne and S. Tringali, The Davenport constant of a box, Acta Arith. 171 (2015), 197-219.

[25] D. R. Richman. Invariants of finite groups over fields of characteristic p, Adv. Math. 124 (1996), 25-48.

[26] B.J. Schmid, Finite groups and invariant theory, Topics in Invariant Theory, Lecture Notes in Mathematics, vol. 1478, Springer, 1991, pp. 35-66.

MTA Alfréd Rényi Institute of Mathematics, ReÁltanoda utca 13-15, 1053 Budapest, HUNGARY

E-mail address: domokos.matyas@renyi.mta.hu 